\title{
ON THE IDEMPOTENCE AND STABILITY OF KERNEL FUNCTORS
}

\author{
by MARK L. TEPLY
}

(Received 6 April, 1993)

1. Introduction. A kernel functor (equivalently, a left exact torsion preradical) is a left exact subfunctor of the identity on the category $R$-mod of left $R$-modules over a ring $R$ with identity. A kernel functor is said to be idempotent if, in addition, $\sigma$ satisfies $\sigma(M / \sigma(M))=0$ for every $M \in R$-mod. To every kernel functor $\sigma$ there corresponds a unique topologizing filter $\mathscr{L}_{\sigma}=\{I \mid \sigma(R / I)=R / I\}$ of left ideals and a unique class $\mathscr{T}_{\sigma}=\{M \in R-\bmod \mid \sigma(M)=M\}$ that is closed under homomorphic images, submodules, and direct sums. The idempotence of $\sigma$ is characterized by either of the following additional conditions:

(1) if $I \in \mathscr{L}_{\sigma}, K \subseteq I$, and $(K: x)=\{r \in R \mid r x \in K\} \in \mathscr{L}_{\sigma}$ for each $x \in I$, then $K \in \mathscr{L}_{\sigma}$; or

(2) $\mathscr{T}_{\sigma}$ is closed under extensions of one member of $\mathscr{T}_{\sigma}$ by another member of $\mathscr{T}_{\sigma}$. Idempotent kernel functors are important since they are the tool used to construct localization functors. For $M \in R-\bmod$, let $E(M)$ denote the injective hull of $M$. A kernel functor $\sigma$ is called stable if $M \in \mathscr{T}_{\sigma}$ implies that $E(M) \in \mathscr{T}_{\sigma}$. For more information about kernel functors, see [6], [7], [14], and [15].

New uses for the kernel functor ([6], [12], [13]) and the questions raised in the recent paper [14] have sparked new interest in the problem of determining when every kernel functor for $R$-mod is idempotent. In particular, must $R$ be left noetherian?

In this paper, we extend some of the results in [4], [6], [10], and [15] by showing that the questions about the idempotence center around the stability of the Gabriel kernel functor. In Theorem 3 we show that when every kernel functor for $R$-mod is idempotent, $R=R_{1} \oplus R_{2}$ (ring direct sum), where $R_{1}$ is a ring with Gabriel dimension and $R_{2}$ has no nonzero ideals with Gabriel dimension. This separates the idempotence question into the study of rings with Gabriel dimension and rings without Gabriel dimension. In Theorem 4 , we show that if $R$ has Gabriel dimension and every kernel functor for $R$-mod is idempotent, then $R$ is left noetherian. In Theorem 7 we give several characterizations of when a ring with Gabriel dimension satisfies the condition: every kernel functor for $R$-mod is idempotent. One of the equivalent conditions is that every kernel functor for $R$-mod is stable. This property has independent interest in view of the paper [11] by Papp and subsequent papers by Papp and others (e.g., see [3] and [5, Chapter 50]) on the question: when is every idempotent kernel functor stable? Finally, in the spirit of $[\mathbf{1 5}$, Theorem 2.6], we give two decomposition theorems for rings satisfying the conditions:

(1) every kernel functor for $R$-mod is stable, or

(2) every kernel functor for $R$-mod is idempotent and the Gabriel kernel functor $\mu$ is stable.

If every kernel functor for $R$-mod is idempotent, it is well known ([6] or [14]) and easy to prove that $R$ is a left nonsingular semiprime ring such that $I^{2}=I$ for every two-sided ideal $I$ of $R$.

Let G-dim $M$ denote the Gabriel dimension of a left $R$-module $M$ (when it exists). 
We will make extensive use of the Gabriel (idempotent) kernel functor $\mu$ : for $M \in R-\bmod , \mu(M)=M$ if and only if G-dim $M$ exists. For a nonlimit ordinal $\alpha$, a nonzero module $M$ is called $\alpha$-simple if $\mathrm{G}-\operatorname{dim} M=\alpha$, but $\mathrm{G}-\operatorname{dim}(M / N)<\alpha$ for every nonzero submodule $N$ of $M$. If G-dim $M$ exists for a nonzero module $M$, then $M$ contains an $\alpha$-simple module for some nonlimit ordinal $\alpha>0$. The 1 -simple modules are precisely the usual simple $R$-modules. A nonzero submodule of an $\alpha$-simple module is $\alpha$-simple. If $R$ has Gabriel dimension, so does every left $R$-module. For the relevant definitions and more information on the Gabriel dimension, see [8] or [9].

2. The results. We will make use of the following standard construction of a kernel functor.

Lemma 1. Let $\mathscr{C}$ be a class of modules, and let $\sigma_{\mathscr{C}}(M)=M$ if and only if $M$ is a homomorphic image of a submodule of a direct sum of modules in $\mathscr{C}$. Then $\sigma_{\mathscr{G}}$ is a kernel functor.

Proof. It is easy to check that the class $\mathscr{T}=\left\{M \mid \sigma_{\mathscr{G}}(M)=M\right\}$ is closed under homomorphic images, submodules, and direct sums; hence $\sigma_{\mathscr{C}}$ must be a kernel functor.

Note that the class $\mathscr{T}$ in the proof of Lemma 1 is the smallest class associated with a kernel functor such that $\mathscr{C} \subseteq \mathscr{T}$. We adopt the notation $\sigma_{\mathscr{G}}$ from Lemma 1 as standard for later use in the paper.

The following lemma about the Gabriel kernel functor $\mu$ is basic for our results.

Lemma 2. Suppose that every kernel functor for $R \cdot \bmod$ is idempotent. If $M$ is a semisimple module, then $\mu(E(M))=M$.

Proof. Let $M$ be a semisimple module, and suppose that $M \subsetneq \mu(E(M))$. Then there exists $N \subseteq E(M)$ such that $N / M$ is $\alpha$-simple for some nonlimit ordinal $\alpha>0$. Further, since Soc is an idempotent kernel functor by hypothesis, then $\alpha \neq 1$. Let $\mathscr{C}$ be the class of all 1 -simple and $\alpha$-simple modules. By Lemma 1 we can define a kernel functor by $\sigma_{\mathscr{C}}(W)=W$ if and only if $W$ is a homomorphic image of a submodule of a direct sum of modules in $\mathscr{C}$. Since $\sigma_{\mathscr{C}}$ is idempotent by hypothesis, then $\sigma_{\mathscr{C}}(N)=N$.

Let $\theta: K \rightarrow N$ be an epimorphism, where $A=\underset{\gamma \in I}{\oplus} A_{\gamma}$ with each $A_{\gamma}$ 1-simple, $B=\bigoplus_{\gamma \in J} B_{\gamma}$ with each $B_{\gamma} \alpha$-simple, and $K \subseteq A \oplus B$. Since $\theta^{-1}(M)$ is essential in $K$, we may use Zorn's Lemma to choose an essential submodule ${ }_{R} H$ of $A \oplus B$ maximal with respect to $H \cap K=\theta^{-1}(M)$. Then

$$
N / M \cong K / \theta^{-1}(M) \rightarrow(A \oplus B) / H
$$

is an embedding. Since $H$ is essential in $A \oplus B$, then $H \cap A_{\gamma}=A_{\gamma}$ for each $\gamma \in I$ and $H \cap B_{\gamma} \neq 0$ for each $\gamma \in J$. Thus

$$
\begin{aligned}
\alpha & =\mathrm{G}-\operatorname{dim}(N / M) \\
& \leq \mathrm{G}-\operatorname{dim}(A \oplus B) / H \\
& \leq \mathrm{G}-\operatorname{dim}\left((A \oplus B) /\left[\left(\bigoplus_{\gamma \in I} H \cap A_{\gamma}\right) \oplus\left(\bigoplus_{\gamma \in J}^{\oplus} H \cap B_{\gamma}\right)\right]=\mathrm{G} \cdot \operatorname{dim}\left(\bigoplus_{\gamma \in J}\left(B_{\gamma} / H \cap B_{\gamma}\right)\right)<\alpha,\right.
\end{aligned}
$$


as each $B_{\gamma}$ is $\alpha$-simple and $\alpha$ is not a limit ordinal. This gives a contradiction; so $M=\mu(E(M))$, as desired.

We now use Lemma 2 to separate the study of rings for which every kernel functor is idempotent into the study of rings with G-dimension and rings in which no nonzero ideal has G-dimension. We note that if $R=R_{1} \oplus R_{2}$ is a ring direct sum and if $M$ is an $R_{1}$-module, then $M$ is naturally an $R$-module such that $\mathrm{G}$-dim $\left({ }_{R} M\right)=\mathrm{G}-\operatorname{dim}\left({ }_{R_{1}} M\right)$ when either side exists. In particular, $\mathrm{G}-\operatorname{dim}\left({ }_{R} R_{1}\right)=\mathrm{G}-\operatorname{dim}\left({ }_{R_{1}} R_{1}\right)$ when either side exists.

THEOREM 3. Suppose that every kernel functor for $R$-mod is idempotent. Then $R=R_{1} \oplus R_{2}$ (ring direct sum), where $R_{1}$ has Gabriel dimension and no nonzero ideal of $R_{2}$ has Gabriel dimension.

Proof. We need to show that $\mu(R)$ is a ring direct summand of $R$. By Zorn's Lemma, choose a two-sided ideal $M$ maximal with respect to $\mu(R) \cap M=0$. By Lemma 1 we can define a kernel functor $\sigma$ via $\sigma(N)=N$ if and only if $N$ is a homomorphic image of a submodule of a direct sum of copies of $\mu(R) \oplus(R /(\mu(R) \oplus M))$. Since $\sigma$ is idempotent by hypothesis, then $\sigma(R / M)=R / M$. Hence there is an epimorphism $\theta: K \rightarrow R / M$, where $K \subseteq A \oplus B, A=\oplus \mu(R)$, and $B=\oplus R /(\mu(R) \oplus M)$.

Let $L=K \cap B$, and let

$$
H / M=\sum\left\{f(L) \mid f \in \operatorname{Hom}_{R}(L, R / M)\right\}
$$

Then $H / M$ is a two-sided ideal of $R / M$, and $\mu(R)(H / M)=\mu(R) \sum f(L)=\sum f(\mu(R) L)=$ 0 , as $\mu(R) B=0$.

If $H / M \neq 0$, then by our choice of $M, \mu(R) \cap H \neq 0$. Since every two-sided ideal of $R$ is idempotent via our hypothesis, then $\mu(R)(H / M) \supseteq(\mu(R) \cap H)[((\mu(R) \cap H) \oplus M) / M]=$ $((\mu(R) \cap H) \oplus M) / M \neq 0$, which contradicts the previous paragraph. Hence $H / M=0$.

Now $\theta(L) \subseteq H / M=0$ : so $\theta$ induces an epimorphism

$$
\theta^{\prime}: K / L \rightarrow R / M \text {. }
$$

Let

$$
\text { - } \pi: A \oplus(B / L) \rightarrow A
$$

be the projection map. Since $(K / L) \cap(B / L)=0$ by the definition of $L$ and since $K / L \subseteq A \oplus(B / L)$, then the restriction of $\pi$ to $K / L$ is a monomorphism.

Assume that $R /(\mu(R) \oplus M) \neq 0$. Let $P$ be a maximal left ideal of $R$ such that $\mu(R) \oplus M \subseteq P$, and let

$$
\eta: R /(\mu(R) \oplus M) \rightarrow R / P
$$

be the natural epimorphism. Then we obtain an epimorphism

$$
\eta \theta^{\prime} \pi^{-1}: \pi(K / L) \rightarrow R / P
$$


Since $\pi(K / L) \subseteq A$, we can extend $\eta \theta^{\prime} \pi^{-1}$ to a homomorphism

$$
\psi: A \rightarrow E(R / P)
$$

by injectivity. Since $\mu(A)=A$, then $\psi(A)=\psi(\mu(A)) \subseteq \mu(E(R / P))=R / P$ by Lemma 2 . But im $\eta \theta^{\prime} \pi^{-1}=R / P$; so $\psi(A)=R / P$. Since $(\mu(R))^{2}=\mu(R)$ via our hypothesis, then $R / P=\psi(A)=\psi(\oplus \mu(R))=\mu(R) \psi(\oplus \mu(R))=\mu(R)(R / P)=0$, as $\mu(R)$ is a two-sided ideal contained in $P$. This contradicts our choice of $P$; hence $R=\mu(R) \oplus M$, as desired.

Viola-Prioli [14] (see also Handelman [10]) has conjectured that if every kernel functor for $R$-mod is idempotent, then $R$ is left noetherian. Our next result shows that the conjecture is true for rings with Gabriel dimension.

THEOREM 4. Assume that every kernel functor for $R$-mod is idempotent. Then the following statements are equivalent.

(1) $R$ has Gabriel dimension.

(2) $\mu$ is a stable kernel functor for $R$-mod.

(3) $R$ is left noetherian.

Proof. (1) $\Rightarrow(2)$ is trivial.

(2) $\Rightarrow(3)$. Let $M$ be a semisimple module. By (2) and Lemma 2, we have $E(M)=\mu(E(M))=M$; hence every semisimple module is injective. Thus $R$ is left noetherian by [2, Proposition 1].

(3) $\Rightarrow(1)$. Left noetherian rings always have Gabriel dimension. (See [8] or [9].)

We want to characterize the rings with Gabriel dimension such that every kernel functor is idempotent. To do this we need two more lemmas.

In [15] Viola-Prioli considers the strong conditions, " $\sigma(M)$ is injective for every nontrivial $\sigma$ " and "every $\sigma$ splits", to obtain results about the condition "every kernel functor $\sigma$ for $R$-mod is idempotent". The condition that $\sigma$ splits means that $\sigma(M)$ is a direct summand of every $R$-module $M$; in particular, $\sigma(E)$ is a direct summand of each injective module $E$. But the splitting by $\sigma$ of injective modules is well-known to be equivalent to the condition, " $\sigma$ is stable". Hence the following lemma of Bican, Jambor, Kepka, and Nemec explains the underlying cause for the success of much of the work in [15].

LEMMA 5 ([1] or [6, Ex. 5.4]). If a kernel functor $\sigma$ is stable, then $\sigma$ is idempotent.

If $E$ is a uniform module, then the sum of $\beta$-simple submodules of $E$ is a $\beta$-simple submodule. Consequently, if we consider the kernel functor $\sigma_{\mathscr{C}}$ of Lemma 1 with $\mathscr{C}=$ all $\alpha$-simple and $\beta$-simple modules $\}$ for fixed $\beta \leq \alpha$, the proof of Lemma 2 shows mutatis mutandis the following result.

Lemma 6. Suppose that every kernel functor for $R$-mod is idempotent. If $M$ is a $\beta$-simple module $(\beta>0)$, then $\mu(E(M))$ is $\beta$-simple.

THEOREM 7. Let $R$ be a ring with Gabriel dimension. Then the following statements are equivalent.

(1) Every kernel functor for $R$-mod is idempotent.

(2) Every kernel functor for $R$-mod is stable.

(3) (i) $R$ is left noetherian, and

(ii) if $C$ is a uniform cyclic $R$-module and $N$ is a nonzero submodule of $C$, then $\sigma_{\{N\}}(C)=C$. 
(4) (i) $R$ is left noetherian, and

(ii) if $I$ is a left ideal such that $I \subsetneq J$ and $I \subsetneq K$ imply that $I \subsetneq J \cap K$, then for any $x \in R-I$ there exist $r_{1}, r_{2}, \ldots, r_{n(x)} \in R$ such that $\bigcap_{i=1}^{n(x)}\left(I: r_{i} x\right) \subseteq I$.

Proof. (1) $\Rightarrow(3) . R$ is left noetherian by Theorem 4 .

Let $C$ be a uniform cyclic $R$-module and $N$ an $R$-module such that $0 \neq N \subseteq C$, and $\sigma_{\{N\}}=\sigma$. We show $\sigma(C)=C$. Since $R$ has Gabriel dimension, $N$ contains a nonzero $\beta$-simple module $S$ for some ordinal $\beta$. By Lemma 6 and the fact that $R$ has Gabriel dimension, we have $E(N)=\mu(E(N))=\mu(E(S))$ is $\beta$-simple for some ordinal $\beta>0$. Hence $C$ must be $\beta$-simple.

Let $\mathscr{C}=\{\sigma(C) \oplus(C / \sigma(C))\}$. By (1) $\sigma_{\mathscr{C}}$ is idempotent, and hence $\sigma_{\mathscr{C}}(C)=C$. Let $\theta: K \rightarrow C$ be an epimorphism, where $K \subseteq A \oplus B, A=\oplus \sigma(C)$, and $B=\oplus(C / \sigma(C))$. Let $L=K \cap B$. Since $\sigma(C)$ is essential in $C$, then $\mathrm{G}-\operatorname{dim}(C / \sigma(C))<\beta$ and hence $\mathrm{G}-\operatorname{dim}(L)<\beta$. Since $C$ is $\beta$-simple, we must have $\theta(L)=0$. Hence $\theta$ induces an epimorphism

$$
\theta^{\prime}: K / L \rightarrow C
$$

Let $\rho: A \oplus B \rightarrow A \oplus(B / L)$ be the natural epimorphism and let $\pi: \rho(A \oplus B) \rightarrow A$ be the natural projection. Then the restriction of $\pi$ to $\rho(K)$ is a monomorphism. Hence $K / L=\rho(K) \cong \pi \rho(K) \subseteq A=\oplus \sigma(C)$ and

$$
\theta^{\prime}: \rho(K) \rightarrow C
$$

is an epimorphism. Since $\sigma(A)=A$, then $\sigma(\rho(K))=\rho(K)$, and hence $\sigma(C)=C$, as desired.

(3) $\Rightarrow(2)$. Let $\tau$ be a kernel functor, and let $\tau(M)=M \neq 0$. Show that $\tau(E(M))=$ $E(M)$. Since $R$ is left noetherian, $E(M)=\underset{\gamma}{\oplus} E_{\gamma}$, where each $E_{\gamma}$ is an indecomposable injective module.

Let $x \in E_{\gamma}$, and let $N$ be a nonzero submodule of $M \cap R x$. Since $N \subseteq M$ and $\tau(M)=M$, then $\sigma_{\{N\}}(L) \subseteq \tau(L)$ for every left $R$-module $L$. But $\sigma_{\{N\}}(R x)=R x$ by (3); so $\tau(R x)=R x$. Since $x$ was arbitrarily chosen in $E_{\gamma}$, then $\tau\left(E_{\gamma}\right)=E_{\gamma}$.

Therefore, $\tau(E(M))=\tau\left(\underset{\gamma}{\oplus} E_{\gamma}\right)=\underset{\gamma}{\oplus} \tau\left(E_{\gamma}\right)=\underset{\gamma}{\oplus} E_{\gamma}=E(M)$.

(2) $\Rightarrow(1)$ is immediate from Lemma 5 . ideals.

$(3) \Leftrightarrow(4)$. This is merely a translation of terminology about cyclic modules and left

Many papers (e.g., see [3], [11], and [5, Chapter 50]) have considered the problem of determining when every idempotent kernel functor is stable. Theorem 7 clearly has a close relationship with the results of these papers, which are lacking in ideal theoretic characterizations for stability in the general case.

While Theorem 7 gives a complete characterization for the stability and idempotence of kernel functors for a ring with Gabriel dimension, the ideal theoretic condition may not always be easy to use to check practical examples. Consequently, we give two decomposition theorems that are in the spirit of [15, Theorem 2.6]. These decomposition 
theorems may make it easier to spot rings in which every kernel functor is stable or idempotent. We recall that $R$ is a left $V$-ring if every simple left $R$-module is injective.

THEOREM 8. Every kernel functor for $R-\bmod$ is stable if and only if $R=\bigoplus_{i=1}^{n} R_{i}$, where each $R_{i}$ is a simple left noetherian, left $V$-ring such that every kernel functor for $R_{i}$-mod is stable.

Proof. $(\Leftarrow)$. Trivial.

$\Leftrightarrow)$. Since every kernel functor for $R$-mod is stable, then every kernel functor is idempotent by Lemma 5 . Hence $R$ is left noetherian by Theorem 4 . Now $R$ is a left $V$-ring by [4, Proposition 6], [6, Proposition 5.29], or Lemma 2.

It is now sufficient to show that $R$ is a direct sum of simple rings. But we can use the argument of Theorem 3 to show that each two-sided ideal $I$ of $R$ is a ring direct summand of $R$. (Just replace $\mu(R)$ by $I$ in the proof and use the fact that $R$ is a left $V$-ring to get im $\psi=R / P$ near the end of the proof.) Consequently, it follows from standard arguments that $R$ is a direct sum of simple rings.

THEOREM 9. Every kernel functor for $R$-mod is idempotent and $\mu$ is stable if and only if $R \cong \bigoplus_{i=1}^{n} R_{i}$, where each $R_{i}$ is a simple left noetherian, left $V$-ring such that every kernel functor for $R_{i}$-mod is idempotent.

Proof. This is an easy modification of the proof of Theorem 8 .

In [10] Handelman shows that if every kernel functor for $R$-mod is idempotent, then $R$ is a subdirect product of strongly prime rings. Theorem 9 shows that we can make a considerable gain in structure by assuming that $\mu$ is stable for $R$-mod.

\section{REFERENCES}

1. L. Bican, P. Jambor, T. Kepka and P. Němec, Stable and costable preradicals, Acta Univ. Carolinae-Math. et Phys. 16 (1975), 63-69.

2. K. A. Byrd, Rings whose quasi-injective modules are injective, Proc. Amer. Math. Soc. 33 (1972), 235-240.

3. R. F. Damiano and Z. Papp, On consequences of stability, Comm. Algebra 9 (1981), 747-764.

4. M. H. Fenrick, Conditions under which all preradical classes are hereditary torsion classes, Comm. Algebra 2 (1974), 365-376.

5. J. S. Golan, Torsion theories, Pitman Monographs and Surveys in Pure and Applied Mathematics 29 (Longman Scientific and Technical, 1986).

6. J. S. Golan, Linear topologies on a ring: an overview, Pitman Research Notes in Mathematics Series 159 (Longman Scientific and Technical, 1987).

7. O. Goldman, Rings and modules of quotients, J. Algebra 13 (1969), 10-47.

8. R. Gordon and J. C. Robson, Krull dimension, Mem. Amer. Math. Soc. 133 (American Mathematical Society, 1973). $459-473$.

9. R. Gordon and J. C. Robson, The Gabriel dimension of a module, J. Algebra 29 (1974)

10. D. Handelman, Strongly semiprime rings, Pacific J. Math. 60 (1975), 115-122.

11. Z. Papp, On stable noetherian rings, Trans. Amer. Math. Soc. 213 (1975), 107-114. 
12. A. M. D. Viola-Prioli and J. E. Viola-Prioli, Rings whose kernel functors are linearly ordered, Pacific J. Math. 132 (1988), 21-34.

13. A. M. D. Viola-Prioli and J. E. Viola-Prioli, Asymmetry in the lattice of kernel functors, Glasgow Math. J. 33 (1991), 95-97.

14. A. M. D. Viola-Prioli and J. E. Viola-Prioli, Rings arising from conditions on preradicals, Proceedings of the XXIst Ohio State/Denison Conference (World Scientific Publishing, 1993), 343-349.

15. J. E. Viola-Prioli, When is every kernel functor idempotent?, Canad. J. Math. 27 (1975), 545-554.

Department of Mathematical Sciences

UNIVERSITY OF WisConsIN-MilWAUKEe

MiLWAUKEE WI 53201,

U.S.A. 